\title{
Percepção dos riscos e vitimização de presos do estado do Rio de Janeiro
}

\author{
Perception of risks and victimization of inmates in the state of Rio de Janeiro \\ Percepción de riesgos y victimización de internos en el estado de Rio de Janeiro
}

Recebido: 14/12/2021 | Revisado: 20/12/2021 | Aceito: 24/12/2021 | Publicado: 27/12/2021

\author{
Paulo Henrique Mauricio de Melo \\ ORCID: https://orcid.org/0000-0002-3935-4645 \\ Escola Nacional de Saúde Pública Sergio Arouca, Brasil \\ E-mail: phmeloy@gmail.com
}

\begin{abstract}
Resumo
O presente artigo tem como objetivo investigar as condições de saúde e de vida relacionadas à percepção de risco e vitimização de presos sentenciados e custodiados do sistema prisional do Estado do Rio de Janeiro. A metodologia baseou-se nas informações do Estudo das Condições de Saúde e Qualidade de Vida dos Presos e das Condições Ambientais das Unidades Prisionais do Estado do Rio de Janeiro, desenvolvido pelo Departamento de Estudos de Violência e Saúde Jorge Careli, da Escola Nacional de Saúde Pública Sergio Arouca, da Fundação Oswaldo Cruz, realizado em 2012. Definiu-se a percepção de risco e a vitimização como variáveis dicotômicas. Para estudar os fatores associados ao risco e à vitimização foram utilizados modelos logísticos e foi incorporado o desenho amostral utilizado no estudo. O modelo final para o desfecho sobre a sensação de correr risco foi composto pelas variáveis: cor/raça, frequência das visitas, ser tratado de forma inferior por funcionários do presídio por conta de sua condição social, ter filhos, situação conjugal e faixa de idade. Já o modelo final para o desfecho sobre ter sofrido algum tipo de agressão no interior do presídio apresentou as seguintes variáveis: sexo, escolaridade, idade, ser tratado de forma inferior por funcionários do presídio por conta de sua condição de detento, ser tratado de forma inferior por outros detentos do presídio por conta do tipo de crime cometido e cor/raça. Conclui-se, portanto, que há diferença entre as variáveis que compõem e são determinantes para os dois modelos.
\end{abstract}

Palavras-chave: Risco; Vitimização; Presídio; Modelo Logístico; Amostra Complexa.

\begin{abstract}
This article aims to investigate the health and life conditions related to the perception of risk and victimization of sentenced inmates in custody of the prison system in the State of Rio de Janeiro. The methodology was based on information from the Study of the Health Conditions and Quality of Life of Prisoners and the Environmental Conditions of Prison Units in the State of Rio de Janeiro, developed by the Department of Violence and Health Studies Jorge Careli, of the Escola Nacional de Saúde Pública Sergio Arouca, from the Oswaldo Cruz Foundation, held in 2012. Perception of risk and victimization were defined as dichotomous variables. To study the factors associated with risk and victimization, logistic models were used and the sampling design used in the study was incorporated. The final model for the outcome on the feeling of being at risk was composed of the variables: color/race, frequency of visits, being treated inferiorly by prison employees because of their social status, having children, marital status and age range. The final model for the outcome of having suffered some type of aggression inside the prison had the following variables: gender, education, age, being treated in a lesser way by prison employees because of their condition of inmate, being treated in a way lower by other prison inmates on account of the type of crime committed and color/race. Therefore, it is concluded that there is a difference between the variables that compose and are decisive for the two models.
\end{abstract}

Keywords: Risk; Victimization; Prison; Logistic Model; Complex Sample.

\section{Resumen}

Este artículo tiene como objetivo investigar las condiciones de salud y vida relacionadas con la percepción de riesgo y victimización de los reclusos condenados en custodia del sistema penitenciario del Estado de Río de Janeiro. La metodología se basó en información del Estudio de las Condiciones de Salud y Calidad de Vida de los Reclusos y las Condiciones Ambientales de las Unidades Penitenciarias del Estado de Rio de Janeiro, desarrollado por el Departamento de Estudios de Violencia y Salud Jorge Careli, de la Escola Nacional. de Saúde Pública Sergio Arouca, de la Fundación Oswaldo Cruz, realizado en 2012. Percepción de riesgo y victimización fueron definidas como variables dicotómicas. Para estudiar los factores asociados al riesgo y victimización se utilizaron modelos logísticos y se incorporó el diseño muestral utilizado en el estudio. El modelo final para el desenlace sobre la sensación de estar en riesgo estuvo compuesto por las variables: color / raza, frecuencia de visitas, ser tratado de manera inferior por los 
empleados del centro por su condición social, tener hijos, estado civil y rango de edad. El modelo final para el desenlace de haber sufrido algún tipo de agresión dentro del centro penitenciario tuvo las siguientes variables: sexo, educación, edad, ser tratado de forma menor por los empleados del centro por su condición de recluso, ser tratado de forma inferior por parte de los funcionarios del centro penitenciario. otros presos por el tipo de delito cometido y el color / raza. Por tanto, se concluye que existe una diferencia entre las variables que componen y son determinantes para los dos modelos.

Palabras clave: Riesgo; Victimización; Prisión; Modelo Logístico; Muestra Compleja.

\section{Introdução}

O foco desse estudo recai não somente sobre o risco real, mas também sobre a percepção de risco de pessoas presas em instituições de segurança do Estado do Rio de Janeiro pelo cometimento de diferentes tipos de crimes, com o intuito de compreender o tema investigado num ambiente fechado ao mundo externo, caracterizado pela rígida disciplina e permeado por violências (Almeida, 2012), uma vez que o sistema penitenciário brasileiro apresenta problemas como: falta de respeito aos presos, carência de tratamento médico e atividades físicas periódicas e superpopulação (Almeida, 2005). Está claro que os presídios não proporcionam condições para a ressocialização e, muito menos, recuperação dos presos. O sistema penitenciário falido é reflexo da crise do Governo e da própria sociedade (Almeida, 2005).

Da mesma forma que a violência real, a percepção de risco afeta diretamente a qualidade de vida e o comportamento dos indivíduos (ISP, 2007), podendo ocasionar a diminuição do uso de espaços públicos como parques e praças, aumentando a segregação entre setores da população através de condomínios e shoppings fechados, bem como provocar patologias de natureza psicológica (Rodrigues, Fernandes, 2005). Estas questões sempre estiveram no centro das preocupações dos indivíduos, dado as restrições e as perdas que impõem aos vitimados e aos residentes de áreas com criminalidade (Moura, Silveira Neto, 2012). O crescimento do sentimento de temor dos indivíduos convive com a existência de um enfraquecimento do nível de confiança do cidadão comum com relação às instituições encarregadas de zelar pela segurança pública e punir os responsáveis por algum descumprimento da lei (Morais, 2009).

Os meios punitivos são utilizados desde a Idade Média, onde o corpo era o principal alvo da repressão penal, com a aplicação de castigos corporais que geralmente resultavam na morte do condenado (Foucault, 1990). Somente no fim do século XVIII, através da influência das ideias iluministas e de ideais liberais em alguns países, como França, Inglaterra e Estados Unidos, algumas leis e códigos criminais começaram a ser reformulados, considerando o elemento humano, que passaria a influenciar todas as penas e a constituição do direito penal. A partir desse período começaram o desenvolvimento e as reflexões para construção do sistema penitenciário (Almeida, 2012).

O sistema prisional passou por inúmeras alterações ao longo do tempo nas diversas vertentes (as regras, direitos e deveres, princípios que embasam o ordenamento social, entre outros) que tratam da vida de um ser humano que cometeu um erro ou um descumprimento a regra da época. Entretanto, é imprescindível, que se atente que no momento em que o indivíduo perde a liberdade (mesmo que parcialmente), o mesmo continua a ter direitos estabelecidos mundialmente, intrínsecos do ser humano, tais como a dignidade da pessoa humana, manutenção dos laços afetivos com os seus familiares, o que é de suma importância para a ressocialização e reconstrução da vida do apenado. (Dullius, Hartmann, 2011)

A ONU, através de inúmeros Congressos, propôs um consenso geral de princípios e práticas no tratamento de reclusos e na gestão dos estabelecimentos de detenção. Reconhecendo-se que há grande variedade de condições legais, sociais, econômicas e geográficas no mundo, espera-se que nem todas as regras tenham condições de serem aplicadas, porém, estas servem como estímulo para que as dificuldades práticas de suas aplicações sejam superadas, uma vez que representam, no seu conjunto, as condições mínimas aceitas como adequadas pelas Nações Unidas (UNODC 2010). 
No nosso país, inicialmente, as políticas para punição eram baseadas em ordenações manuelinas e filipinas , que tinham como base a ideia de utilizar o terror para intimidação e com isso criar um instrumento punitivo contra o crime, empregando ideias políticas e religiosas da época (Dullius, Hartmann, 2011). Já em 1830, após a independência, iniciou-se a construção de uma legislação própria à população brasileira, se adequando, principalmente, a afastar o domínio e a opressão dos colonizadores. (Cuano, 2001)

Em 1890 foi criado um Código Penal, baseado no princípio da utilidade pública, trazendo inúmeros avanços e mudanças como a individualização e o princípio da utilidade da pena, a abolição da pena de morte e o surgimento de um regime penitenciário de caráter correcional, com finalidade de reeducar o detento para sua reinserção na sociedade.

Durante o governo de Getúlio Vargas, em 1940, foi publicada a consolidação das Leis Penais, o que foi chamado de Código Penal Brasileiro, e a partir desse momento as penas foram divididas em principais e acessórias, dependendo da gravidade do delito cometido, sendo as principais classificadas em: reclusão, detenção e multa; e as acessórias em: perda da função pública, interdições de direitos e publicação da sentença. Esse modelo penal de 1940 sofreu mudanças em 1969, 1977, 1981 e 1984, sempre se adequando à ideologia da época. No ano de 1984, estabeleceu-se a Lei da Execução das Penas (Lei 7210), que visa regulamentar a classificação e individualização das penas, definindo normas básicas para tratamento do apenado, resguardando os direitos e estabelecendo os deveres. Com a Constituição da República Federativa do Brasil de 1988 foram incorporadas várias matérias pré-estabelecidas, atendo-se principalmente, ao princípio da humanidade como por exemplo a proibição da tortura e respeito à integridade física e moral, significando, neste ponto, um grande avanço no sistema democrático Brasileiro (Cuano, 2001).

No Rio de Janeiro, com o passar do tempo, o Sistema Penitenciário sofreu mudanças significativas no seu regulamento, funcionamento e disciplina, influenciadas pelo aumento da população carcerária. Esse aumento fez com que se iniciasse um processo de expansão no início da década de 1940, como por exemplo a inauguração das primeiras unidades em Bangu. Após a mudança da capital federal para Brasília em 1960, o modelo utilizado durante anos, com penitenciárias sendo alvo de elogios quanto as suas instalações físicas, disciplina e segurança, começa a perder "fôlego". Com a diminuição de investimentos e melhorias, inicia-se uma crise, dificultando ao Sistema Penitenciário, já estadualizado, manter o nível existente nos anos anteriores. Apesar de aumentar o número das unidades e de internos, não se mudou a visão da prisão pela sociedade como sendo um depósito de presos que não cumpre o papel de formador de mão de obra (Almeida, 2012).

\section{Metodologia}

\subsection{Modelo de Regressão Linear}

Um modelo de regressão linear é um método onde se utiliza a relação entre duas ou mais variáveis para descrever o comportamento de um fenômeno. Chama-se de variável resposta a variável que se deseja explicar e de variáveis preditas ou explicativas as que irão contribuir para a explicação da variável resposta (Dobson, Barnett, 2008).

O modelo de regressão linear clássico é definido como:

$Y_{i}=\beta_{i} X_{i}+\varepsilon_{i}$

Onde,

$Y_{i}$ é o vetor correspondente a variável resposta com distribuição $N\left(\mu_{i}, \sigma^{2}\right)$;

$\beta_{i}$ é o vetor correspondente aos parâmetros, desconhecidos, e que são estimados, geralmente, pelo método de mínimos quadrados; 
$X_{i}$ é o vetor correspondente à(s) variável(is) explicativa(s);

$\varepsilon_{i}$ é o vetor correspondente aos erros aleatórios e independentes com distribuição $N\left(0, \sigma^{2}\right)$ sendo $\sigma^{2}$ constante.

Temos que,

$E\left(Y_{i}\right)=\mu_{i}=\beta_{i} X_{i}$

\subsection{Família Exponencial}

Inúmeras distribuições estatísticas conhecidas podem ser reunidas na chamada família exponencial univariada. Dentre elas, citamos as distribuições Normal, Binomial, Binomial Negativa, Gama e Poisson. Essa classe de família de distribuições foi proposta por Koopman, Pitman e Darmois, através da averiguação de propriedades de suficiência estatística. Posteriormente, outros aspectos dessa família foram descobertos e tornaram-se importantes na teoria moderna de Estatística. O conceito de família exponencial foi introduzido na Estatística por Fisher em 1934 (Dobson, Barnett, 2008).

\section{Definição}

Seja X uma variável aleatória cuja função de probabilidade (discreta) ou função de densidade de probabilidade (contínua) dependa de um único parâmetro $\tau$ e a família $\tau=\{\mathrm{f}(\mathrm{x} ; \tau), \tau \in \Omega \subseteq \Re\}$ de f.d.p’́s, diz-se que ela é a família exponencial de distribuições com parâmetro $\tau$ se:

$f(x ; \tau)=\exp [q(x) s(\tau)+\ln t(\tau)+\ln h(x)]$

E tomando-se $d(\tau)=\ln t(\tau)$ e $g(x)=\operatorname{lnh}(x)$, teremos:

$f(x ; \tau)=\exp [q(x) s(\tau)+d(\tau)+g(x)] I_{A}(x)$

Desde que q(.) e s(.) sejam funções monotônicas, pode-se fazer:

$s(\tau)=\frac{\theta}{a(\phi)}$ e $q(X)=Y$,

sendo $\phi>0$, conhecido e fixo, temos então,

$f(y ; \theta)=\exp \left\{\frac{1}{a(\phi)}\left[y \theta+d_{1}(\theta)\right]+g_{1}(y ; \phi)\right\}$

Em que $d_{1}($.$) e g_{1}($.$) São funções conhecidas.$

Na notação de McCullagh \& Nelder (1983), teremos,

$f(y ; \theta, \phi)=\exp \left\{\frac{1}{a(\phi)}[y \theta-b(\theta)]-c(y ; \phi)\right\} I_{A}(y)$ 
Para $b($.$) e c(.) funções conhecidas e \phi>0$, suposto conhecido.

Caso $\phi$ seja desconhecido, $\mathrm{f}(\mathrm{y} ; \boldsymbol{\theta}, \phi)$ poderá pertencer ou não à família exponencial com dois parâmetros.

Ao fazermos $\phi=\frac{1}{\mathrm{a}(\phi)}$, temos a notação usada por Cordeiro (1986), isto é:

\section{$f\left(y ; \theta, \phi^{\prime}\right)=\exp \left\{\phi^{\prime}\left[y \theta-b(\theta)+c_{1}\left(y ; \phi^{\prime}\right)\right]\right\} I_{A}(y)$,}

Sendo $c_{1}($.$) conhecida.$

Tem-se que, nestes últimos dois casos, a família exponencial está na forma canônica com um único parâmetro (canônico ou natural) $\theta$. Se existirem outros parâmetros além de $\theta$, eles serão vistos como parâmetros de perturbação (muisance parameters).

\subsection{Modelos Lineares Generalizados}

De acordo com Dobson e Barnett (2008), o MLG pode ser definido como um grupo de modelos estatísticos que permitem analisar a relação entre várias características referentes a um grupo de sujeitos ou objetos. Estes modelos procuram explicar como varia a média de uma (ou mais) variável(eis) resposta(s), ou dependente(s), em função de variáveis explicativas ou preditoras.

Os MLG constituem uma extensão dos modelos lineares de regressão múltipla e sua passagem, da versão clássica, para os MLG permitiu alargar as hipóteses admitidas. A variável resposta do modelo passa a poder provir de um universo que siga uma lei de distribuição da família exponencial, deixando de ter obrigatoriamente uma distribuição Normal. Além disso, a passagem dos modelos lineares de regressão clássicos para os MLG contém outra generalização. Se nos primeiros a relação entre o valor médio da variável resposta e a combinação linear das variáveis explicativas é a função identidade, nos segundos aquela relação pode ser estabelecida por qualquer função monótona e diferenciável (Demétrio, 2002).

Um MLG pode ser caracterizado nos seguintes pontos (McCullagh, Nelder, 1989):

Considerem-se $\mathrm{N}$ variáveis aleatórias $\mathrm{Y}_{\mathrm{i}}(\mathrm{i}=1, \ldots, \mathrm{N})$ independentes, de média $\mu_{\mathrm{i}}$ respectivamente e função de probabilidade ou função de densidade de probabilidade pertencente à família exponencial, isto é:

$$
f\left(y_{i} / \theta_{i, \emptyset}\right)=\left\{\frac{y_{i} \theta_{i}-b\left(\theta_{i}\right)}{a_{i}}+c\left(y_{i}, \emptyset\right)\right\}
$$

Onde $a(),. b($.$) e c(.) são funções específicas para cada distribuição da família exponencial com parâmetro canônico \theta$. Caso $\theta$ seja desconhecido, a distribuição pode não pertencer à família exponencial e há de se considerar somente os casos em que a distribuição pertence àquela família.

Geralmente, a função $a_{i}(\emptyset)$ toma a seguinte forma:

$$
a_{i}(\emptyset)=\frac{\emptyset}{w_{i}}
$$

Onde se assume que $w_{\mathrm{i}}$ é conhecido e $\emptyset$, denominado parâmetro de dispersão ou de escala, pode ser ou não. 
Note-se que, em um MLG, a distribuição de todas as variáveis aleatórias $Y_{\mathrm{i}}$ é da mesma forma (Normal, Poisson, Gama ou outra da família exponencial) e o parâmetro de escala, $\phi$, é constante, ou seja, não varia com o índice i das variáveis aleatórias $Y_{\mathrm{i}}$.

Desse modo, fica definida a componente aleatória do modelo.

Suponha-se agora a existência de $\mathrm{p}$ variáveis explicativas $\mathrm{X}_{\mathrm{j}}(\mathrm{j}=1, \ldots, \mathrm{p})$.

As $\mathrm{N}$ observações destas p variaveis constituem a matriz $\mathrm{X}$. Isto é, cada elemento $\mathrm{X}_{\mathrm{ij}}$ da matriz $\mathrm{X}$ designa o valor da $\mathrm{j}$ ésima variável explicativa para a observação $\mathrm{i}(\mathrm{i}=1, \ldots, \mathrm{N})$.

A partir desta matriz define-se um preditor linear $\eta_{\mathrm{i}}(\mathrm{i}=1, \ldots, \mathrm{N})$, da forma:

$$
\eta_{i}=\sum_{j-1}^{p} x_{i j} \beta_{j}
$$

Constituindo os $\beta_{\mathrm{j}}(\mathrm{j}=1, \ldots, \mathrm{p})$, um vetor de parâmetros desconhecidos, a estimar a partir dos dados.

$\mathrm{Na}$ forma matricial, temos:

$\vec{\eta}=x \vec{\beta}$

Onde seus componentes são:

i) Um vetor de parâmetros $\beta=\left(\beta_{1}, \ldots, \beta_{p}\right)$ e as variáveis explicativas $X$;

ii) Um preditor linear $\eta_{i}$ que envolve as variáveis regressoras ou covariáveis;

iii) A função de ligação $\mathrm{g}\left(\right.$.) que relaciona o preditor linear $\eta_{i}$ com a média natural da variável resposta. Se a função de ligação for escolhida de forma errada, podem ocorrer sérios problemas no modelo.

McCullagh e Nelder (1989) apresentam quatro funções utilizadas na modelagem de dados cuja variável é binária:

1. Função logística;

2. Função probito;

3. Função complementar log-log;

4. Função log-log.

Assim fica especificada a componente sistemática.

As duas componentes anteriores relacionam-se através de uma função de ligação $g($.$) , que se admite existir, ser$ monótona e diferenciável, e que transforma $\mu_{\mathrm{i}}$ em $\eta_{\mathrm{i}}$, ou seja:

$\eta_{i}=g\left(\mu_{i}\right), i=1, \ldots, N$

Para construirmos um MLG, deve-se: 
i) Definir a variável resposta e as variáveis explicativas;

ii) Definir a distribuição de probabilidade da variável resposta;

iii) Definir a função de ligação.

Ficam assim definidas as componentes de um modelo linear generalizado.

\subsection{Teste de Wald}

Em seguida à estimação dos parâmetros, deve-se proceder à investigação da significância estatística dos mesmos. O teste de Wald é utilizado para avaliar, individualmente, se o parâmetro é estatisticamente significativo. A estatística de teste utilizada é obtida através da razão do coeficiente pelo seu respectivo erro padrão.

Para testar se os coeficientes apresentam efeitos semelhantes sobre o modelo, utilizasse a Estatística de Wald, e esta é baseada na distribuição marginal assintoticamente normal dos parâmetros.

As hipóteses a serem testadas são as seguintes:

$$
H_{0}: \beta_{i}-\beta_{j}=0
$$

$H_{1}: \beta_{i}-\beta_{j} \neq 0$

Sendo $\beta_{\mathrm{i}}$ e $\beta_{\mathrm{j}}$ parâmetros do modelo, tal que $\mathrm{i} \neq \mathrm{j}$.

Utiliza-se a estatística de teste:

$W=\frac{\left(\hat{\beta}_{i}-\hat{\beta}_{j}\right)}{\sqrt{\hat{V}\left(\hat{\beta}_{i}-\hat{\beta}_{j}\right)}}$

Que sob a hipótese descrita acima, tem distribuição assintoticamente Normal Padrão - N(0,1).

Sendo,

$$
\begin{aligned}
& \hat{V}\left(\hat{\beta}_{i}-\hat{\beta}_{j}\right)=\hat{V}\left(\hat{\beta}_{i}\right)+\hat{V}\left(\hat{\beta}_{j}\right)-2 \operatorname{cov}\left(\hat{\beta}_{i}, \hat{\beta}_{j}\right) \\
& \widehat{\operatorname{cov}}\left(\hat{\beta}_{i}, \hat{\beta}_{j}\right)=\operatorname{cor}\left(\hat{\beta}_{i}, \hat{\beta}_{j}\right) * \sqrt{\hat{\beta}_{i}} * \sqrt{\hat{\beta}_{j}}
\end{aligned}
$$

Na qual,

$\hat{V}\left(\hat{\beta}_{i}\right)$ é a variância estimada do estimador $\hat{\beta}_{i}$.

$\hat{V}\left(\hat{\beta}_{j}\right)$ é a variância estimada do estimador $\hat{\beta}_{j}$.

$\widehat{\operatorname{cov}}\left(\hat{\beta}_{i}, \hat{\beta}_{j}\right)$ é a covariância estimada dos estimadores $\hat{\beta}_{i}$ e $\hat{\beta}_{j}$. 


\subsection{Modelo Teórico}

Para tal estudo serão utilizados dois modelos de regressão logísticos, que tem por característica a análise de dados onde a variável resposta qualitativa é dicotômica, por exemplo, sim ou não, 0 ou 1. O modelo de regressão logístico pode ser definido como uma equação matemática em que se expressa o relacionamento de variáveis, definindo-se uma variável dependente, ou variável resposta, e verificando-se a influência de uma ou mais variáveis ditas variáveis independentes ou explicativas sobre esta variável dependente (Zanini, 2007). Uma das grandes vantagens da regressão logística é que cada coeficiente estimado fornece uma estimativa do logaritmo natural do odds ratio ajustado para todas as variáveis do modelo, permitindo a estimação direta do odds ratio através da exponenciação do coeficiente $\beta_{\mathrm{i}}$.

No caso desse estudo, a variável dependente do primeiro modelo é dada pela composição de uma pergunta da pesquisa que se refere à sensação de risco sofrida pelo detento, ou seja, as situações pelas quais ele acha que corre o risco de passar dentro do presídio, tendo como opções: sofrer agressão física, sofrer violência sexual (assédio, estupro), sofrer violência psicológica (ameaças, humilhações), ser ferido(a) por arma branca, ser ferido(a) por arma de fogo, queimadura por fogo ou química, explosão (bomba, granada, outros explosivos) e outros; Sendo assim, a variável dependente será uma variável dicotômica de forma que o detento assinalou pelo menos uma das opções, ela assumirá o valor 1, caso nenhuma opção seja assinalada, ela assumirá o valor 0. Portanto, tem-se uma variável que poderá assumir somente os valores 0 e 1 , enquanto a pergunta que originou a variável resposta possui oito opções de resposta e é permitida a marcação de nenhuma ou mais de uma opção.

A variável dependente do segundo modelo é dada pela composição de uma pergunta da pesquisa que se refere às situações sofridas pelo detento, tendo como opções: sofrer agressão física, sofrer agressão verbal, sofrer violência sexual (assédio, estupro), perfuração por arma de fogo, perfuração por arma branca, queda, tentativa de suicídio, tentativa de homicídio;

As possíveis variáveis explicativas para os dois modelos são as que descrevem as características socioeconômicas do indivíduo (tais como sexo, idade, cor de pele, escolaridade), a situação da sua vida fora da instituição (situação conjugal, se possui filhos, como está o contato com a família, se recebe e qual a frequência das visitas), as situações recorrentes do preso no seu ambiente de convívio (se já foi tratado de forma inferior por outros detentos ou por funcionários, se já sofreu agressão, etc.) e as referentes à saúde do indivíduo (prática de atividades físicas e se possui lesões permanente e se estas foram causadas após o ingresso no presídio). Desta forma, será possível averiguar e identificar quais os principais fatores que influenciam e estão associados à percepção de risco e vitimização do grupo de presos sentenciados e custodiados do estado do Rio de Janeiro, em cada um dos modelos. A metodologia deu-se por iniciar com o modelo completo, ou seja, com todas as variáveis escolhidas, e a partir desse modelo verificar a necessidade de retirar alguma variável ou fazer algum ajuste de categoria.

Ainda há que ser ressaltada a necessidade de incluir o desenho amostral da pesquisa, pois a pesquisa utilizada como base para o projeto e a maioria dos inquéritos nacionais que levantam informações de saúde como, por exemplo, a Pesquisa Nacional por Amostra de Domicílios (PNAD), a Pesquisa Nacional de Saúde do Escolar (PeNSE), realizadas pelo Instituto Brasileiro de Geografia e Estatística (IBGE), e a Pesquisa Mundial de Saúde (PMS 2003), realizada pela Organização PanAmericana da Saúde (OPAS), utilizam planos de amostragem complexa (Silva et al., 2002).

Para efetuar a análise de dados provenientes de inquéritos amostrais complexos é preciso incorporar as informações do plano amostral da pesquisa, informando os pesos das unidades da amostra e as informações estruturais da pesquisa, identificando o estrato e pelo menos as unidades primárias de amostragem, uma vez que as estimativas pontuais são influenciadas por pesos distintos associados às unidades da amostra, enquanto as estimativas de variância são influenciadas pela estratificação, conglomeração e pesos amostrais. Portanto, um modelo linear generalizado de dados coletados através de 
uma amostragem complexa deve incluir os pesos amostrais e o plano amostral para obter estimativas e variâncias válidas (Szwarcwald, Damacena, 2008).

\section{Resultados}

Após a análise descritiva, foram definidas as variáveis que serão adicionadas aos modelos finais. As variáveis selecionadas anteriormente foram analisadas separadamente e incorporadas aos modelos apenas com as respectivas variáveis de desfecho, com o objetivo de analisar o comportamento junto as variáveis de estudo para analisar as Odds Ratio - OR brutas sem a influência das demais variáveis. Essas associações individuais foram feitas com o objetivo de identificar quais variáveis seriam adequadas para serem incorporadas ao modelo. Ou seja, o processo se inicia com o modelo nulo de cada variável resposta, cada uma sendo explicada somente pela componente aleatória, posteriormente são incluídas as variáveis explicativas que, individualmente, foram significativas na variação da resposta. Após a inclusão de todas as variáveis, a decisão passa a ser de qual variável não é estatisticamente significativa (através do teste de Wald) para variável resposta considerando que as demais variáveis influenciam nessa relação. A cada passo, uma variável é retirada e o novo modelo é analisado até chegar em um conjunto de variáveis onde não há mais variáveis a serem retiradas. Esse processo é conhecido como método de seleção backward.

\section{a) Sentimento de risco}

A variável idade permitia a possibilidade de ser usada como variável contínua, como variável contínua com componente quadrático ou de forma categórica. Foi verificado que ao adicionar a componente quadrática retira-se a representatividade da componente linear, como não foi encontrada nenhuma evidência de associação da componente quadrática com o desfecho, a opção foi não usar a componente quadrática, portanto a variável idade foi analisada em forma de categorias, o que também facilita a interpretação das razões de chances.

Outra variável que poderia sofrer alteração nas suas categorias é a variável cor ou raça, porém a categoria pardo apresentou-se protetora e a categoria amarela ou indígena, de risco melhorando a explicação das demais categorias quando colocadas na base e tornando a análise mais detalhada, logo, esta variável também teve sua configuração mantida.

Para o primeiro modelo, onde a variável resposta é a percepção de risco, foram selecionadas as seguintes variáveis: sexo, faixa de idade, raça/cor, frequência das visitas, ter sido tratado de forma inferior pelos funcionários pela condição de detento, cor de pele, condição social e tipo de crime e ter sido tratado de forma inferior por outros detentos pela condição de detento, cor da pele, condição social e orientação sexual.

Considerando a relação da variável resposta do primeiro modelo com cada uma das variáveis escolhidas e o intercepto, verifica-se que algumas apresentam p-valor relativamente alto e em algumas categorias a odds ratio (OR) não deve ser interpretada uma vez que o intervalo de confiança contém o valor 1. Porém, todas as variáveis descritas serão analisadas conjuntamente, assim como suas respectivas interações. Após a análise conjunta, a retirada da primeira variável será realizada levando em consideração o maior p-valor para o teste de Wald e as correlações entre as variáveis, uma vez que variáveis muito correlacionadas com a variável dependente e com uma ou mais variáveis independentes, podem gerar confusão se analisada conjuntamente num modelo (Luiz, 2002).

O modelo iniciou-se com a variável resposta, percepção de risco no interior do sistema prisional, sendo explicada pelas variáveis: sexo, faixa de idade, raça/cor, frequência das visitas, ter sido tratado de forma inferior pelos funcionários pela condição de detento, cor de pele, condição social e tipo de crime e ter sido tratado de forma inferior por outros detentos pela condição de detento, cor da pele, condição social e orientação sexual. 
Considerando as etapas enunciadas anteriormente, obteve-se um modelo parcimonioso com pseudo R2 de 0,492, ou seja, com as variáveis explicativas escolhidas, aproximadamente $50 \%$ da variação da variável resposta está sendo explicada.

O modelo final para o desfecho sobre a sensação de correr risco foi composto pelas variáveis: cor/raça, ser tratado de forma inferior por funcionários do presídio por conta de sua condição social, possuir filhos e faixa de idade.

$\mathrm{Na}$ cor ou raça, a categoria preta não foi significativa ao nível de 5\%, não permitindo interpretar seu resultado. Porém, pode-se verificar que em relação à categoria referente aos pardos (base), os brancos têm aproximadamente cinco vezes as chances de acharem que correm risco no interior do presídio, enquanto que para a categoria amarela/indígena essa relação é maior que cinquenta vezes. Essas conclusões são válidas na condição das demais variáveis que compõem o modelo se manterem constantes.

Os presos que não foram tratados de forma inferior por funcionários no interior do presídio têm praticamente $96 \%$ menos chance de sentir que correm risco do que os que sofreram esse tratamento, se todas as outras covariáveis forem mantidas constantes.

O fato de não ter filho (s) aparenta ser um fator de "proteção" uma vez que ter filhos aumenta em 357,5\% a chance de se sentir inseguro dentro da prisão em relação à quem não tem filho (s).

A única comparação possível de ser feita com a variável idade é a da faixa de 25 a 29 anos apresentar quase 15 vezes a chance de se sentir em risco em relação à faixa de 18 a 24 anos, mantendo todas as variáveis constantes.

Foi avaliada também a entrada de componente de interação entre as variáveis explicativas. A interação que proporcionou maior ganho na explicação do modelo, fazendo o pseudo R2 sair de 0,492 para 0,657 foi a inclusão da relação entre a idade e a cor ou raça. Outras combinações de interações não apresentaram melhoras significativas. Apesar de melhorar a explicação do modelo, a inclusão da interação fez com que o intervalo de variação e o próprio valor das Odds ratio, na maioria dos coeficientes das variáveis explicativas, ficassem enormes, o que faria com que estes não pudessem ser utilizados para compor o conjunto de variáveis que explicam a variação do desfecho. Com isso, optou-se em não incluir o termo de interação.

\section{b) Vitimização}

Assim como no primeiro modelo, a variável idade foi investigada e chegou-se à conclusão que sua forma composta de faixas, tornando-a categórica, era a melhor maneira de inseri-la na análise. A variável cor ou raça também apresentou a característica protetora e a de risco para a cor parda e amarela ou indígena respectivamente.

Para o segundo modelo, onde a variável resposta é o fato de ter sofrido algum tipo de vitimização, foram selecionadas as seguintes variáveis: faixa de idade, raça/cor, escolaridade, ter sido tratado de forma inferior pelos funcionários pela condição de detento, cor de pele e tipo de crime e ter sido tratado de forma inferior por outros detentos pelo tipo de crime e aparência física.

Considerando a relação da variável resposta do segundo modelo com cada uma das variáveis escolhidas e o intercepto, verifica-se, assim como no primeiro modelo, que algumas apresentam p-valor relativamente alto e em algumas categorias a odds ratio não devem ser interpretadas uma vez que o intervalo de confiança contém o valor 1 . O critério de manter todas as variáveis descritas para serem analisadas conjuntamente, assim como suas respectivas interações será mantido. Após a análise conjunta, a retirada ou não das variáveis será pelo critério da parcimônia, melhor poder de explicação e base teórica.

No segundo modelo foram adicionadas as respectivas covariáveis determinadas através dos resultados descritivos e da análise dos modelos bivariados para determinar a explicação da variável resposta ter sofrido algum tipo de agressão. 
O método utilizado consistiu em iniciar pelo modelo completo, com todas as variáveis escolhidas, e retirar, uma a uma, as variáveis que não forem significativas para a explicar a variação da variável resposta. Com isso, o modelo iniciou-se com a variável resposta, vitimização (ter sofrido algum tipo de agressão) no interior do sistema prisional, sendo explicada pelas variáveis: sexo, faixa de idade, raça/cor, escolaridade, ter sido tratado de forma inferior pelos funcionários pela condição de detento, cor de pele e tipo de crime e ter sido tratado de forma inferior por outros detentos pelo tipo de crime e pela aparência física.

As variáveis explicativas escolhidas, depois de cumpridas todas as etapas enunciadas anteriormente, formaram um modelo parcimonioso com pseudo $\mathrm{R}^{2}$ de 0,584 , ou seja, aproximadamente $60 \%$ da variação da variável resposta estão sendo explicada.

O modelo final para o desfecho sobre ter sofrido algum tipo de agressão no interior do presídio apresentou as seguintes variáveis: sexo, escolaridade, idade, ser tratado de forma inferior por funcionários do presídio por conta de sua condição de detento, ser tratado de forma inferior por outros detentos do presídio por conta do tipo de crime cometido e cor/raça.

Mantendo as demais variáveis constantes, as mulheres apresentam uma chance quase dezoito vezes maior de ter sofrido agressão em relação aos homens.

A única comparação possível de ser feita com a variável escolaridade é a da faixa fundamental até a $4^{\mathrm{a}}$ série apresentar 37 vezes mais de chance de ter sofrido agressão em relação à faixa acima de ensino médio completo, mantendo todas as variáveis constantes.

Assim como a escolaridade, a variável idade apresenta somente uma única comparação possível de ser feita com a faixa 25 a 29 anos, que possui 9,4 vezes a chance de ter sofrido agressão em relação à faixa de 18 a 24 anos, mantendo todas as variáveis constantes.

A chance de não ser tratado de forma inferior por parte dos funcionários por conta da condição de detento, é 5,4 vezes a chance de ser tratado de forma inferior. Quando o tratamento por conta do tipo de crime é feito por outros detentos, a chance de ter passado por algum tipo de situação relacionado a agressões é seis vezes a chance de não ter passado. Em ambos os casos as bases são as situações de não terem recebido o respectivo tratamento e para que as comparações sejam válidas, as demais variáveis não podem ser alteradas.

A variável cor ou raça teve a categoria preto/pardo não significativa ao nível de 5\%, não permitindo interpretar seus resultados. A única categoria possível de ser interpretada é a de quem está classificado na categoria amarela/indígena tem mais de vinte e sete vezes a chance de ter sofrido uma agressão no interior da prisão essa relação a quem está classificado como branco e mantendo as demais variáveis, que compõe o modelo, constantes.

Foi realizado o teste para a entrada da componente de interação entre as variáveis explicativas. Assim como no primeiro modelo, a única interação que proporcionou um ganho significativo na explicação do modelo, fazendo o pseudo $\mathrm{R}^{2}$ sair de 0,584 para 0,726 foi novamente a inclusão da relação entre a idade e a cor ou raça. Outras combinações de interações não apresentaram melhoras significativas. Mesmo melhorando a explicação do modelo, a inclusão da interação no segundo modelo também foi rejeitada pelos mesmos motivos da rejeição no modelo anterior: tornar boa parte da análise dos coeficientes das variáveis explicativas inconclusivas, pois faz "explodir” as Odds Ratio.

\section{Discussão}

O significado da palavra violência vem do latim violentia e significa a força que se usa contra o direito e a lei. Com o passar do tempo, passou a ser empregada com o sentido de ruptura da ordem ou emprego de algum artifício para impor uma 
ordem, significando assim, o emprego de força ou dominação sem legitimidade. No caso de força por parte do Estado, mesmo em alguma situação em que esse uso seja legítimo, ele não pode violar a vida do cidadão, sendo ele presidiário ou não, uma vez que as autoridades públicas devem zelar pela vida quando alguma ação é tomada e coloca em risco a vida de terceiros (Misse, 2006).

Qualquer estudo sobre a população carcerária deve contemplar e se apropriar de estudos sobre a sociedade a qual esse cárcere está ligado, uma vez que estão inseridos numa instituição social, convivendo com alguns cidadãos e tendo contato com outros que não estão necessariamente na condição de presidiários.

No estudo da sociedade atual é necessário abandonar a concepção rígida das estruturas urbanas complexas e se aprofundar nas relações humanas e sociais que existem nesses domínios, gerando cadeias de ideias, medos e expectativas que não se limitam ao espaço que cerca o cidadão, uma vez que essas cadeias se propagam e rompem quaisquer barreiras físicas (Melo, Hanna L. C. F., 2010).

A segregação social e territorial é uma das principais consequências do recente medo de violência, seja ela contra a própria pessoa, pessoas próximas ou contra o patrimônio.

Além da violência aumentando e se tornando cada vez mais um sentimento intrínseco aos seres humanos, há vários outros fatores que contribuem para o crescimento desse medo, assim como a mídia que fertiliza a imaginação e influencia uma vez que dá grande visibilidade aos casos de violência.

Com o aumento da violência, tanto o aumento real quanto o de exposição, eleva-se também o medo dos cidadãos em relação a alguns lugares, seja esse medo real ou imaginário (Melo, 2010).

E o medo é algo que está presente em todos os seres vivos, sendo antes de qualquer coisa, um choque, gerado pela percepção de algum perigo próximo (Mira y López, Lima, 1988). Portanto, o medo não é algo controlável pelo ser, mas sim uma reação decorrente da relação dele com o meio em que vive. Com isso, o ser humano começa a evitar e fugir por causa do medo em si ao invés do objeto que provoca esse sentimento, uma vez que a iminência do fato já é o suficiente pra provocar a reação no corpo e provocar esse "alerta” (Mira y López, Lima, 1988).

Enquanto nos demais animais a interpretação do medo é algo mais fácil de ser entendida, pois está quase que exclusivamente ligada a uma questão de sobrevivência natural pela relação de caça e caçador, no homem essa interpretação se torna mais complexa por questão de processos mutáveis como cultura, sociedade, religião, época, etc ( (Melo, 2010).

Nos últimos tempos, tanto no Brasil quanto no mundo, a violência urbana vem ganhando cada vez mais destaque, em noticiários, jornais e no dia a dia da população. E a parte da população vitimada por conta da crescente violência construiu, muito em função da divulgação por parte da mídia, um perfil para relacionar ao agente causador "do mal”, traçando seu próprio perfil, aumentando as barreiras sociais entre as classes econômicas, uma vez que esse estereótipo é baseado na figura das classes mais pobres (Melo, 2010). A violência pode ser definida, também, pela sociologia, traçando uma relação de exclusão causada pelo capitalismo, onde os cidadãos são rebaixados ao nível de mercadorias ou meros clientes (Carbonari, 2002).

A estrutura da sociedade moderna, em que pobreza e riqueza muitas vezes convivem e usufruem do mesmo espaço, proporciona um aumento na violência através da delinquência, que é gerada pela não inserção de parte da população nas categorias de consumo. Ao mesmo tempo em que há o convívio nas grandes metrópoles, existe a polarização, separando a população mais pobre em bairros decadentes, marginalizando e gerando o perfil de delinquência que a maioria da população atribui à parcela mais pobre da sociedade. Esse exílio em lugares menos privilegiados, onde há maior incidência de violência, está diretamente associado ao processo de sujeição criminal (Misse, 2006). Porém, a violência é um processo sistêmico 
abrangente e deve ser estudado levando em consideração que não está restrito a um determinado local ou grupo social ou oriundo de algum grupo específico, é um processo que atinge toda a população.

Estudos têm mostrado as relações entre o risco percebido e a vitimização, ou seja, entre a percepção e a efetivação da violência. Para ambos os casos, a perspectiva teórica que identifica os fatores que propiciam o surgimento de uma eventual violência podem ser separadas em três grupos: questões sociodemográficas, atividades diárias e características do ambiente em que o indivíduo vive; o que é conhecido também como a teoria das oportunidades (Hindelang et al., 1978). Essa teoria aponta para quatro principais conceitos de determinação do risco: proximidade ao crime, exposição ao crime, atratividade do alvo para o criminoso e a existência de vigilância ou uma capacidade de proteção crime (Clarke, 1997).

Seguindo a perspectiva citada acima se tem que o risco percebido e a vitimização são determinados por algumas características pessoais, atividades diárias e características geográficas. Isso pode ser confirmado através dos resultados obtidos neste estudo, uma vez que a constante relação dos prisioneiros com o crime e demais situações de conflito social estão associadas com: as características sociodemográficas deles, como sexo, educação, situação conjugal, filhos e idade; atividades diárias, como frequência de atividades físicas e contato com a religião; e características ambientais e de convívio, como discriminação sofrida por funcionários ou outros detentos e lesões sofridas.

O presente estudo corrobora com essas afirmações, uma vez que em ambos os modelos, ou seja, tanto na percepção do risco quanto na vitimização, a variável cor ou raça está fortemente associada com os desfechos, refletindo a polarização e marginalização que parte da sociedade sofre.

Outra variável que confirma que a disparidade organizacional da sociedade está diretamente relacionada com o que ocorre no interior dos presídios do Rio de Janeiro, que é o fato do nível de educação apresentar distribuição de proporções diferentes de "agredidos" ao longo de suas categorias. Ou seja, duas das principais variáveis relacionadas à desigualdade social e econômica enfrentada pela sociedade, estão diretamente ligadas à sensação de risco ou à vitimização no interior das prisões.

Apesar de existirem vários estudos diferenciando a percepção de risco da violência efetivada, estudos fazendo essa diferenciação na situação específica dos presídios são bastante escassos e majoritariamente qualitativos. Porém, observando os resultados encontrados e os estudos observados, pode-se dizer que a "comunidade" dentro dos presídios segue alguns parâmetros da sociedade em geral.

A escassez de publicações nacionais, até mesmo qualitativas, sobre a situação dos presídios no Brasil dificulta a obtenção de informações sobre as situações que geram a sensação de risco e a violência no interior dos presídios, ou até mesmo a comparação dos resultados obtidos no Rio de Janeiro com o que ocorre em outros estados. Apesar de o assunto ser de suma importância para segurança e saúde não só dos presidiários, mas da população em geral, nenhuma base de dados pública tem informação que possa ser usada para estudo sobre a segurança e condições de saúde dos detentos. Até mesmo, quando o estudo é sobre a população, são poucos os que tratam especificamente dos temas segurança, risco ou vitimização. Por exemplo, a Pesquisa Nacional por Amostra de Domicílios (PNAD) fez um suplemento específico para esses temas em 2009 (Características da vitimização e do acesso à justiça no Brasil), porém, desde 2009 nenhuma outra pesquisa dessa magnitude foi realizada, aliás, todos os últimos suplementos da PNAD contemplaram somente o tema a respeito do acesso à internet, ou telefonia móvel.

Sugere-se, portanto, a realização de mais pesquisas e estudos voltados para a análise da diferença entre percepção de risco e violência efetivada, tanto em populações carcerárias quanto na sociedade em geral.

Nesse estudo, foi respeitado todo o processo da amostragem realizado pela pesquisa "Estudo das Condições de Saúde e Qualidade de Vida dos Presos e das Condições Ambientais das Unidades Prisionais do Estado do Rio de Janeiro", assim 
como seus estratos de representatividade através da incorporação do desenho amostral, minimizando assim os possíveis erros por conta da subestimação das variâncias.

\section{Conclusão}

Os fatores que influenciam no sentimento de correr risco no interior do presídio, aumentando as chances do presidiário sentir que corre esse risco são: ser da raça ou cor indígena, amarela ou branca, possuir filhos, ter sido tratado de forma inferior por funcionários do presídio por conta da sua condição social, ter de 25 a 29 anos; comparadas com suas respectivas bases: ser da cor parda, não possuir filhos, não ter sido tratado de forma inferior por funcionários do presídio por conta da sua condição social, ter de 18 a 24 anos.

Já as categorias das covariáveis que estão relacionadas com a vitimização na prisão, aumentando a chance do preso ter sofrido essa agressão, são: ser do sexo feminino, ter o ensino fundamental até a $4^{\mathrm{a}}$ série, ter sido tratado de forma inferior por outros presos pelo tipo de crime cometido, ter sido tratado de forma inferior por funcionários do presídio pela condição de detento e ser da cor ou raça amarela ou indígena; comparadas com as respectivas categorias de base: ser do sexo masculino, ter no mínimo o ensino médio completo, nunca ter sido tratado de forma inferior por outro presos pelo tipo de crime cometido, nunca ter sido tratado de forma inferior por funcionários do presídio pela condição de detento e ser da cor parda.

Pode-se observar que a diferença básica entre as variáveis que compõem os dois modelos é que no primeiro, que trata de uma percepção, a maioria das variáveis que tiveram forte relação com o desfecho estão relacionadas à vida do preso fora do presídio, como por exemplo o fato de ter ou não filhos (que ficou no modelo final), ou seja, situações que mexem com o sentimento e que não estão presentes em seu dia a dia. Em contrapartida, o segundo modelo que afere vitimização é baseado em variáveis que tratam de acontecimentos da realidade do detento, como por exemplo, ele ter sido inferiorizado por outros detentos ou funcionários, além também da variável sexo, que foi determinante sobre sofrer algum tipo de agressão e não a respeito da sensação de risco.

A falta de mais estudos e mais pesquisas a respeito do tema torna complexa a produção de algum estudo quantitativo sobre o assunto, uma vez que a base teórica para produzir tal investigação não está tão bem fundamentada. A necessidade de um levantamento de dados específico para o tema torna o assunto, que é de extrema importância para políticas públicas de segurança e saúde, muito pouco explorado na literatura quando a comparação entre o risco percebido e a vitimização é utilizada.

Aumentando o número de estudos onde a comparação entre a sensação de estar em risco e o risco efetivo é abordada, pode-se fundamentar com maior propriedade as razões que levam, por exemplo, a condição de ser do sexo feminino ser significativa para explicar a vitimização e não ser para o sentimento de risco, auxiliando, concomitantemente, os estudos específicos sobre violência sofrida pelas mulheres dentro dos presídios.

Por fim, vale deixar claro que a assistência ao preso e ao internado é dever do Estado, de acordo com os artigos 10 e 11 da Seção I do Capítulo II da Lei de Execução Penal brasileira (Lei 7.210/84), sendo essa assistência material, à saúde, jurídica, educacional, social e religiosa.

\section{Referências}

Almeida, A. C. F. (2005): Prisão: uma discussão oportuna. https://www.direitonet.com.br/artigos/exibir/1992/Prisao-uma-discussao-oportuna

Almeida, G. R. de (2012): O Sistema Prisional no Rio de Janeiro. Um breve relato sobre o sistema prisional no Rio de Janeiro. In Museu do Cárcere.

American Psychological Association (2010): Dicionário de Psicologia Apa.

Assis, R. D. de (2007): A realidade atual do sistema penitenciário brasileiro. In Revista CEJ. 
Barbosa, J. F., Chaves, A. B. P., \& Almeida, S. dos S. de. (2020). Vitimização de policiais militares no Estado do Pará (Brasil) em 2019. Research, Society and Development.

Barros, F. de M. (2008): A Participação da Vítima no Processo Penal, Rio de Janeiro: Lumen Juris.

Bengochea, J. L. P.; Guimarães, L. B.; Gomes, M. L.; \& Abreu, S. R. (2004): A transição de uma polícia de controle para uma polícia cidadã.

Boarccaech, A. (2009): Os eleitos do Cárcere.

Brandão, A. A.; Silva, A. P.; Pinto, A. S.; Borges, D.; Luz, E.; \& Soares, G. et al. (2007): Série Análise Criminal. In Pesquisa de Condições de Vida e Vitimização.

Carbonari, P. C. (2002): Ética da Responsabilidade Solidária. In IFIBE.

Clarke, R. V. G. (1997): Situational crime prevention. Successful case studies. Second edition. Guilderland, N.Y.: Harrow and Heston.

Constantino, P (2001): Entre as escolhas e os riscos possíveis. A inserção das jovens no tráfico de drogas. In Fundação Oswaldo Cruz - Escola Nacional de Saúde Pública.

Cuano, R. P. (2001): História do Direito Penal Brasileiro.

Demétrio, C. G. B. (2002): Modelos Lineares Generalizados em Experimentação.

Dobson, A. J.; \& Barnett, A. G. (2008): An introduction to generalized linear models. 3rd ed. Boca Raton: CRC Press (Chapman \& Hall/CRC texts in statistical science series).

Duarte, M. S.; \& Silva, A. P. da (2007): Pesquisa de condições de vida e vitimização. 1a edição (Coleção Instituto de Segurança Pública. Série Análise criminal, volume 2).

Dullius, Aladio A.; Hartmann, J. A. M. (2011): Análise do sistema prisional brasileiro. In Âmbito Jurídico.

Filho, C. M. (2001): Violência fundadora e violência reativa na cultura brasileira. São Paulo em Perspectiva.

Foucault, M. (1990): Vigiar e Punir. História da violência nas prisões.

Giddens, A. (2000): Mundo na era da globalização. 2. ed. Lisboa: Presença.

Gois, S. M.; Silveira, F. A. M.; \& Gaudêncio, M. P M. (2012): Para além das grades e punições. Uma revisão sistemática sobre a saúde penitenciária.

Hayes, M. V. (1992): On the epistemology of risk: language, logic and social scien-ce. In Social science \& medicine.

Hindelang, M. J.; Gottfredson, M. R.; \& Garofalo, J. (1978): Victims of personal crime: an empirical foundation for a theory of personal victimization. An empirical foundation for a theory of personal victimization. Cambridge, Mass.: Ballinger.

ISP (Ed.) (2007): Pesquisa de Condições de Vida e Vitimização. Instituto de Segurança Pú-blica (Série Análise Criminal, 2).

Krug, E. G.; Dahlberg, L. L.; Zwi, A. B.; \& Lozano, R. (2002): World report on violence and health. Geneva: World Health Organization.

Lagrange, R.; \& Ferraro, K. (1987): The elderly's fear of crime. A critical examination of the research. In Research on Aging.

Luiz, R. R. (2002): Inferência causal em epidemiologia. O modelo de respostas potenciais. Rio de Janeiro: Editora Fiocruz.

McCullagh, P.; \& Nelder, J. A. (1989): Generalized linear models. 2nd ed. London, New York: Chapman and Hall (Monographs on statistics and applied probability, 37).

Melo, H. L. C. F. (2010): A Violência na cidade. Um breve ensaio sobre o estigma territorial.

Michaud, Y. (1989): A violência. In São Paulo: Ática.

Mira y López, E.; \& Lima, C. de A. (1988): Quatro gigantes da alma. O medo, a ira, o amor, o dever. 13. ed. Rio de Janeiro: José Olympio.

Misse, M. (2006): Crime e violência no Brasil contemporâneo. Estudos de sociologia do crime e da violência urbana. Rio de Janeiro: Editora Lumen Juris (Coleção Conflitos, direitos e culturas).

Morais, D. (2009): Padrões de Criminalidade e Espaço Público. o centro do Rio de Janeiro. In Núcleo de Estudos sobre Segurança e Política Criminal (NESPC). 
Moura, K. H. de L.; \& Silveira Neto, R. da M. (2012): Vitimização nos Centros Urbanos Brasileiros. Uma Abordagem Multinível.

Nery, B. B. (2005): O cárcere e seus problemas. https://www.direitonet.com.br/artigos/exibir/2107/O-carcere-e-seus-problemas

Reis, C. B.; \& Bernardes, E. B. (2011): O que acontece atrás das grades. estratégias de preven-ção desenvolvidas nas delegacias civis contra HIV/AIDS e outras doenças sexualmente trans-missíveis.

Rodrigues, C. D.; \& Fernandes, R. A. (2007): Medo do Crime - Percepção ou realidade? Uma Análise Comparada do Risco Percebido e Risco Objetivo de Vitimização Local e Não Local.

Rodrigues, C. D.; \& Fernandes, R. A. (2005): Medo do Crime - Percepção ou realidade? Uma Análise Comparada do Risco Percebido e Risco Objetivo de Vitimização Local e Não Local.

Rountree, P. W.; \& Land, K. (1996): Perceived risk versus fear of crime. Empirical evidence of conceptually distinct reactions in survey data. In Social Forces, 1996 (74), pp. 1353-1376.

Salla, F. (1999): Para uma análise histórica do sistema prisional brasileiro, com destaque para São Paulo. As Prisões em São Paulo 1822-1940.

Schneier, B. (2009): The Psychology of Security, 2009.

Silva, P. L. do N.; Pessoa, D. G. C.; \& Lila, M. F. (2002): Análise estatística de dados da PNAD: incorporando a estrutura do plano amostral. In Ciênc. saúde coletiva.

Silva, G. K. N.; Vedovello, V. P.; \& Fegadolli, C. (2008): Políticas e práticas da assistência farmacêutica penitenciária. In UNIMEP[periódico na Internet]. Skinner, B. F. (1974): About Behaviorism. In Appleton-Century-Crofts.

Sousa, M. da C. P. de; Alencar Neto, F. J. de; \& Silva, C. L. da C. (2013): Atenção à saúde no sistema penitenciário. Revisão de literatura. In Revista Interdisciplinar.

Sousa, F. B. de, Soares, J. de F., Bezerra Neto, F. das C., Caiana, C. R. A., Medeiros, A. C. de, Silva, E. P., França Júnior, R. P., Félix, M. da C. S., Wanderley, H. G. F., \& Maracajá, P. B. (2020). Sistema prisional brasileiro: infraestrutura, rebeliões e administração de crises. Research, Society and Development.

Szwarcwald, C. L.; \& Damacena, G. N. (2008): Amostras complexas em inquéritos populacionais: planejamento e implicações na análise estatística dos dados. In Rev. bras. epidemiol.

Teixeira, P. R (2001): A AIDS nos presídios. In Boletim Direitos Humanos HIV/AIDS - Brasília: Ministério da Saúde, 2001 (1).

UNODC (2010): Congressos das Nações Unidas sobre prevenção ao Crime e Justiça Criminal 1955-2010.

Varela, C (2005): ¿Qué significa estar seguro? De delitos, miedos e inseguridades entre los adultos mayores.

Zanini, A. (2007): Regressão logística e redes neurais artificiais: um problema de estrutura de preferência do consumidor e classificação de perfis de consumo, Juiz de Fora. FEA/UFJF. 